\title{
WAVELET BASED ECG SIGNAL COMPONENT IDENTIFICATION
}

\author{
Tatiparti Padma ${ }^{1}$, Chepuri Bhavana ${ }^{2}$, Peddamallu Sridivya ${ }^{3}$ \\ ${ }^{1}$ Faculty, Department of Biomedical Engineering, GRIET, AP, INDIA \\ ${ }^{2}$ Student, Department of Biomedical Engineering, GRIET, AP, INDIA \\ ${ }^{3}$ Student, Department of Biomedical Engineering, GRIET, AP, INDIA
}

\begin{abstract}
The most commonly used technique to record the bioelectric current generated by the heart is Electrocardiogram. It is a simple, painless and valuable test done by placing the surface electrodes at the predetermined locations on the surface of the heart. It is a composite signal consisting of $P, Q R S, T$ and $U$ waves, vital information related to arrhythmias (heart rhythm abnormalities) can be extracted from intervals and segments of these waves. The present study includes the development of an algorithm which preprocesses and automatically extracts the features of an ECG signal using wavelet transformation. As Wavelets provide both temporal and spatial information simultaneously and offers flexibility with the choice of wavelet functions with different properties. The initial step of the algorithm includes pre-processing of the signal in order to remove noise from the signal (denoising) and then uses the pre-processed signal for feature extraction. The accuracy of the analysis is increased and also the analysis time is reduced by using this algorithm.
\end{abstract}

Keywords - ECG, P wave, QRS complex, T wave, R-R interval, ST interval, QT interval, ST segment, heart rate, Wavelet transform.

\section{INTRODUCTION}

The action potentials generated by all the heart muscle fibers during each heart beat collectively forms ECG record. Electrocardiograph is an instrument used to record the changes in ECG. During diagnosis, electrodes are positioned on the arms and legs (limb leads) and at six positions on the chest (chest leads). The electrocardiograph is capable of amplifying the heart's electrical signals and produces 12 different tracings from various combinations of limb and chest leads. The slight difference in the electrical activity is due to placement of limb and chest electrodes at different positions relative to the heart. By comparing these records with one another and with normal records, it is possible to determine (i) if the conducting pathway is abnormal, (ii) if the heart is enlarged, (iii) if certain regions of the heart are damaged, and (iv) the cause of angina. In a typical record, three distinct waves appear with each heartbeat. The first wave is $\mathrm{P}$ wave, it is a small upward deflection on the ECG. The $\mathrm{P}$ wave represents atrial depolarization. The second wave, is QRS complex, begins as a downward deflection, continues as a large, upright, triangular wave, and ends as a downward wave. The QRS complex represents rapid ventricular depolarization, as the action potential propagates through ventricular contractile fibers. The third wave is a domeshaped upward deflection called the $\mathrm{T}$ wave. It indicates ventricular repolarization. $\mathrm{T}$ wave occurs during the relaxation phase of ventricles. Though it is not always visible, $\mathrm{U}$ wave is considered as the representation of Papillary Muscle or Purkinje Fibers [2]. ECG waveform characteristics and their corresponding positions in heart is shown in the Fig.1 given below. As repolarisation is much slower than depolarization, the $\mathrm{T}$ wave is smaller and wider than the QRS complex. Based on Electrocardiogram signals many of the heart rhythm abnormalities (arrhythmias) can be diagnosed, during this process a physician analyses the signal and extracts the features like amplitudes of the waves and the time interval between them. Larger $\mathrm{P}$ waves indicate enlargement of an atrium, an enlarged $Q$ wave may indicate a myocardial infarction and an enlarged $\mathrm{R}$ wave generally referred as enlarged ventricles [3]. Due to insufficiency of oxygen, the $\mathrm{T}$ wave appears flatter than normal $\mathrm{T}$ wave.

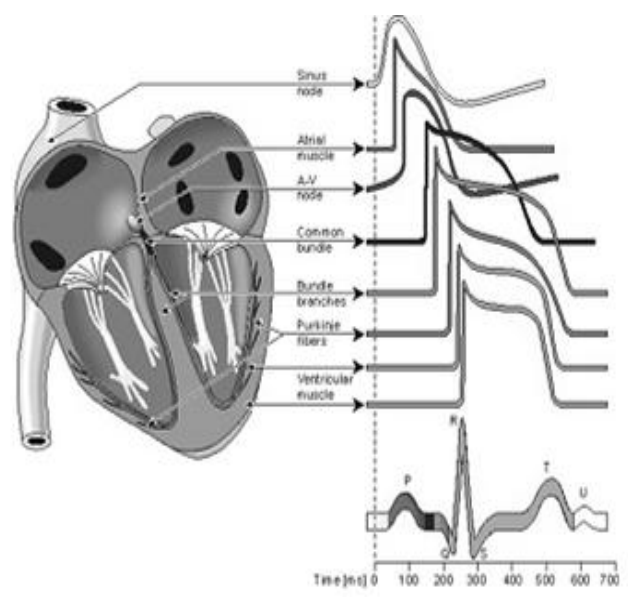

Fig. 1 ECG waveform characteristics and their corresponding positions in heart [1]

For analysis of an ECG intervals or segments (measurement of time between two waves) are to be considered. For example, The Q-T interval extends from the start of the QRS complex to the end of the $\mathrm{T}$ wave. It is the duration from beginning of ventricular depolarization to the end of ventricular repolarization. The $\mathrm{Q}-\mathrm{T}$ interval may be lengthened by myocardial damage, myocardial ischemia (decreased blood flow), or conduction abnormalities. The S-T 
interval is from end of $\mathrm{S}$ to end of $\mathrm{T}$-wave. The normal values of the ECG signal are shown in the Table I below and the typical normal ECG signal is shown in Fig.2. Any change in the given listed values indicates the abnormality of the heart [3].

\section{Table 1}

\begin{tabular}{|l|l|l|}
\hline S.No & Features & Amplitude/Duration \\
\hline 1 & P wave & $0.25 \mathrm{mV}$ \\
\hline 2 & R wave & $1.6 \mathrm{mV}$ \\
\hline 3 & Q-wave & $0.4 \mathrm{mV}$ \\
\hline 4 & T-wave & 0.1 to $0.5 \mathrm{mV}$ \\
\hline 5 & R-R interval & $0.6-1.2 \mathrm{Sec}$ \\
\hline 6 & PR-interval & $0.12-0.2 \mathrm{Sec}$ \\
\hline 7 & QRS complex & 0.04 to $0.12 \mathrm{Sec}$ \\
\hline 8 & S-T interval & $<0.42 \mathrm{Sec}$ \\
\hline 9 & QT interval & $<0.44 \mathrm{Sec}$ \\
\hline 10 & $\begin{array}{l}\text { Normal Heart } \\
\text { Rate }\end{array}$ & $60-100 \mathrm{BPM}$ \\
\hline
\end{tabular}

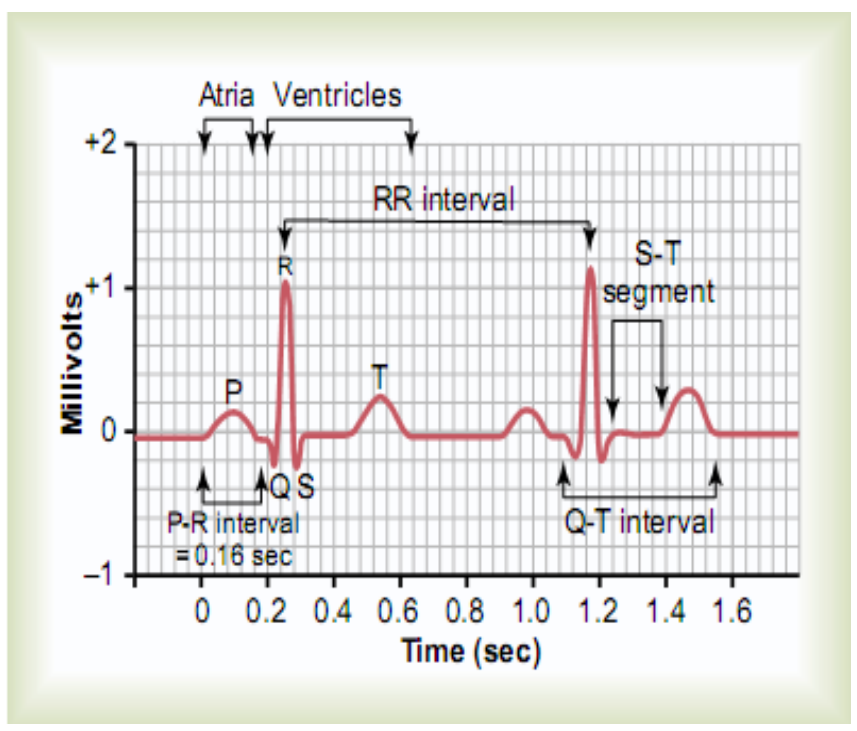

Fig. 2 Normal ECG signal [4]

\section{WAVELET TRANSFORMS}

The mathematical tool for decomposing a signal into a set of orthogonal waveforms both in time and frequency domains is wavelet transform. Wavelets are functions that are used to represent data or other functions and satisfy certain mathematical requirements. In wavelet analysis, the scale used to look at data plays a special role. Morlet first considered wavelets as a family of functions constructed from translations and dilations of a single function called the "mother wavelet" $\psi(\mathrm{t})$. They are defined by

$$
\psi_{\mathrm{a}, \mathrm{b}}(t)=\frac{1}{\sqrt{|a|}} \psi\left(\frac{\mathrm{t}-\mathrm{b}}{\mathrm{a}}\right), \quad \mathrm{a}, \mathrm{b} \in \mathrm{R}, \mathrm{a} \neq \theta
$$

' $\mathrm{a}$ ' is the scaling parameter or scale, and it measures the degree of compression and $b$ is the translation parameter which determines the time location of the wavelet. When $|a|$ $<1$, then the wavelet is in the compressed version (smaller support in time domain) of the mother wavelet and corresponds mainly to higher frequencies. On the other hand, when $|a|>1$, then $\psi_{\mathrm{a}, \mathrm{b}}(\mathrm{t})$ has a larger time-width than $\psi(\mathrm{t})$ and corresponds to lower frequencies. Thus, wavelets have timewidths adapted to their frequencies. This is the main reason for the success of the Morlet wavelets in signal processing and time-frequency signal analysis. Data can be processed at different scales or resolutions using Wavelet algorithms.
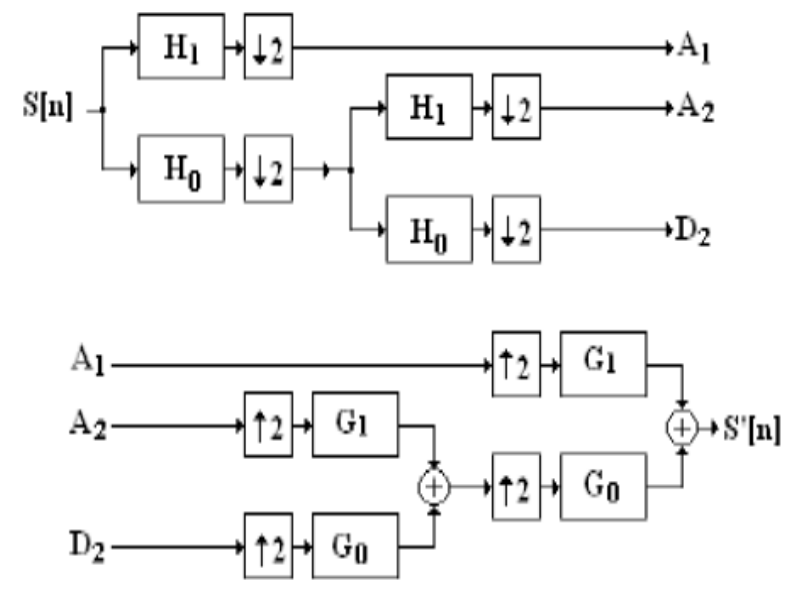

Fig. 3 2nd order DWT decomposition and reconstruction structure [5]

The importance of wavelet analysis is to adopt a wavelet prototype function, called an analysing wavelet or mother wavelet. In the wavelet analysis the filter decomposes the signal into frequency bands. The decomposed signal can be reconstructed to the original bands using filter in wavelet synthesis. Both the shape and perfect reconstruction of wavelet can be determined by selection of filter. Discrete wavelet transform has number of advantages when applied towards ECG analysis. Discrete Wavelet Transform (DWT) has two filters; a Low Pass Filter (LPF) and a High Pass Filter (HPF).They are used to decompose the signal into different scales. The output coefficients of the LPF are called 'Approximation' while the output coefficients of the HPF are called 'Detail'. The Approximation signal can be sent again to the LPF and HPF of the next level for second-level decomposition, thus to decompose the signal into its different components at different scale levels [5]. Decomposition and reconstruction structure of wavelet is shown in Fig. 3.

Some of the wavelet families are Haar, Daubechies, symlets, coiflets etc. Commonly used wavelets for ECG signal processing is Daubechies. In this study, an algorithm is developed to extract the ECG features automatically. The developed algorithm initially decomposes the original ECG signal by using Wavelet Transform and Daubechies wavelet (db4) as mother wavelet. Wavelets are a powerful statistical tool which can be used for a wide range of applications, namely Signal processing, DNA analysis, protein analysis, Speech recognition. Wavelets have been used successfully in other areas of geophysical study. 


\section{FEATURE IDENTIFICATION USING} WAVELETS

The sample ECG signals for the present study are collected from MIT-BIH database from Physionet website and stored in a text format. The MIT-BIH database contains many types of ECG signals including both normal and abnormal, which are sampled at different rates [6]. For example record number 16272 is originally sampled at $128 \mathrm{~Hz}$, record 30 was sampled at $250 \mathrm{~Hz}$ and record 113 was sampled at $360 \mathrm{~Hz}$. Therefore, to process all the signals uniquely, all the samples must be resampled at $360 \mathrm{~Hz}$ before processing the ECG signal. Each selected ECG signal is of thirty minute duration, but only 5 minutes duration of the signal is used for processing in this study.

Discrete Wavelet transform are used to extract features from an electrocardiogram (ECG). Detection can be achieved in a better way by the wavelet filter with scaling function similar to the shape of the ECG signal. The wavelet is similar in shape to QRS complex and their energy spectrum is concentrated around low frequencies excluding the high frequency component and is an approximated ECG signal. Some of the components for analysis of an ECG signal are listed below.

\section{1 $P$ and T-Wave}

Function of atrium and Sino-atrium node (SA) will detect the shape and the time interval of $\mathrm{P}$-wave. It intern will provide the pace to the following components of ECG complex. Thus, a theoretical link between frequency domain spectral analysis techniques and time domain analysis is constructed. The T wave is the positive deflection after each QRS complex. It represents ventricular repolarization. The $\mathrm{T}$ wave contains more information than the QT interval.I

\subsection{QRS Complex}

Most of the energy is concentrated in QRS complex of ECG waveform. It provides reasonably good amount of information about the functioning capability and the pacing ability of human heart. The variation of amplitude or/and time of QRS complex can help in determining the symptoms required for study. QRS interval or duration extends from beginning of $\mathrm{Q}$ to the end of the $\mathrm{S}$ wave. If QRS is greater than $0.12 \mathrm{sec}$, indicates the presence of bundle branch block.

\subsection{ST Segment}

ST segment begins at the end of the $\mathrm{S}$ wave and ends at the beginning of the $\mathrm{T}$ wave. During the plateau phase of the action potential, the ventricular contractile fibers are depolarized that represents the ST segment. In acute myocardial infarction the ST segment is elevated (above the baseline) and depressed (below the baseline) when the heart muscle receives insufficient oxygen.

\subsection{QT, ST and RR Intervals}

The QT interval extends from the beginning of the QRS complex to the end of the $\mathrm{T}$ wave. It is the time duration between ventricular depolarization to ventricular repolarization. The duration of QT interval exceeds (i) in myocardial damage, (ii) myocardial ischemia (decreased blood flow), or conduction abnormalities and (iii) Hypothyroidism. The ST interval is measured from the $\mathbf{J}$ point to the end of the T wave. Junction between the end of QRS and beginning of ST segment is called J point (used to measure degree of ST elevation or depression present). The duration between two consecutive $R$ waves represents $R R$ interval and its normal range is 0.6 to $1.2 \mathrm{sec}$.

\subsection{Heart Rate}

$\mathrm{R}$ Wave is the most dominant wave in ECG complex. The synchronization between the two pace making nodes can be reflected with the normal $\mathrm{R}-\mathrm{R}$ interval. The heart rate can be calculated by successive averaging of time duration between two consecutive $\mathrm{R}$ waves and it is measured in Beats Per Minute (BPM) [1].

\section{PROCEDURE FOR THE PROPOSED}

\section{ANALYSIS}

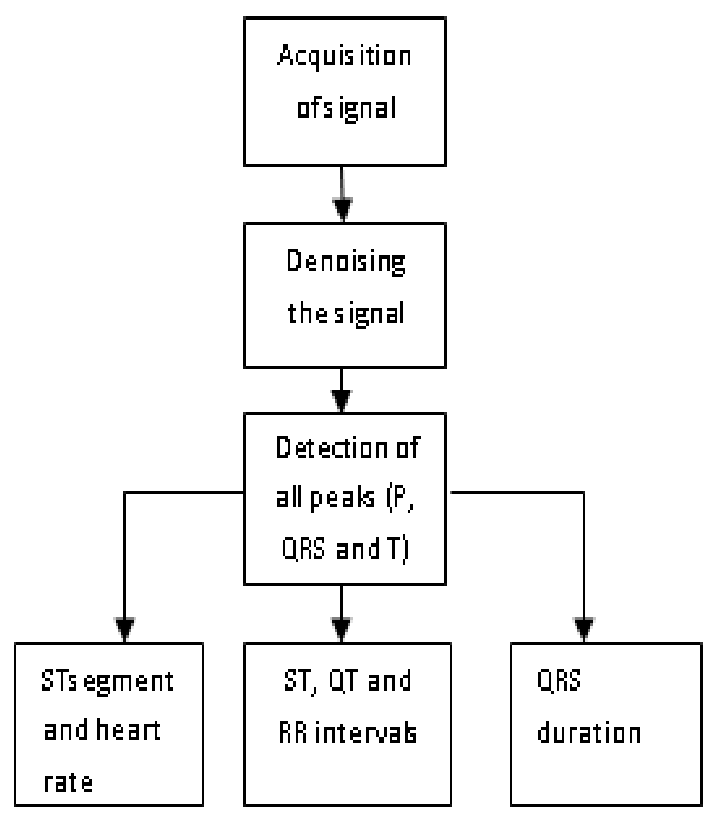

Fig.4 Flow chart for features extraction of ECG signal

The acquired ECG signal is preprocessed to remove noise due to artifacts caused by motion, power line interferences, electrode contact noise, muscle contractions and breath caused baseline movement by decomposing the signal using daubechies (db4) wavelet. The resultant or preprocessed signal is then used to detect all the peaks and then followed by the extraction of above listed parameters. Fig. 4 reflects the flowchart of the developed algorithm. 


\section{EXPERIMENTAL RESULTS}

The algorithm is implemented on various signals of MIT$\mathrm{BIH}$ database. Here 2000 samples of every signal is considered for experimentation. Following are results for the signal 103 is shown in Fig 5(a) and (b).

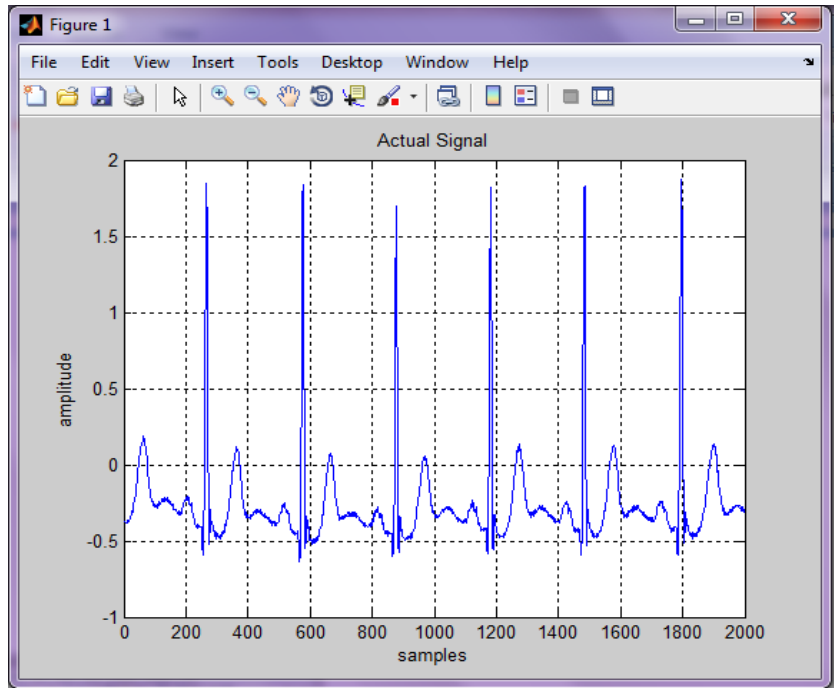

Fig 5(a) Original signal

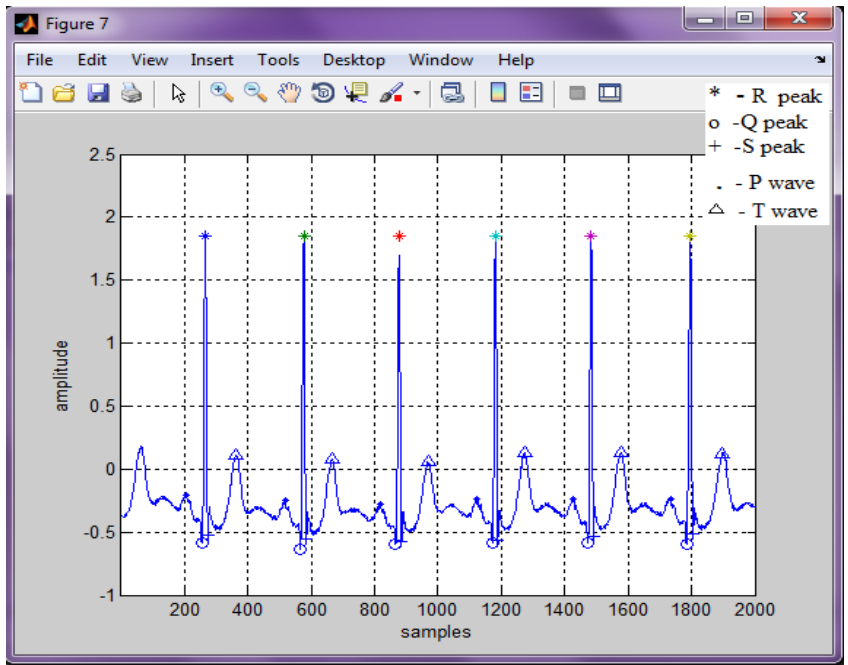

Fig.5 (b) Detected all peaks in ECG signal

From the above detected peaks, the values of ST interval$0.0108 \mathrm{sec}$, QT interval-0.0076sec, RR interval- $0.85 \mathrm{sec}$ and heart rate is 71(normal), QRS duration-0.0254 sec.

\section{CONCLUSIONS AND FUTURE WORK}

The study mainly involves the development of an algorithm that extract the features more accurately. One of the important steps in the ECG analysis is to detect the different waves forming the entire cardiac cycle. Most of the studies based around wavelet transformation identify $99.8 \%$ of ECG waveforms. The wavelet transformation is worth enough in recognition of $\mathrm{P}$ and $\mathrm{T}$ wave.
Wavelet transformation in electro cardiology is relatively new field of research. Many methodological aspects (Choice of the mother wavelet, values of the scale parameters) of the wavelet technique will require further investigations in order to improve the clinical usefulness of this novel signal processing technique. The extracted features can be used further for classification of different heart rhythm abnormalities like tachycardia, Bradycardia and myocardial ischemia etc.

\section{REFERENCES}

[1] J. L. Rajput and K. C S, "Classification of ECG abnormalities using ANN," International Jounral of Research and Technology, vol. 1, no. 5, 2012

[2] B. D. Gerard J. tortora, Principles of Anatomy and Physiology

[3] V. S. Y. N. M. C.Saritha, "ECG Signal Analysis Using Wavelet Transforms," Bulg. J. Phys., vol. 35, pp. 68-77, 2008

[4] C. Guyton and J. E. Hall, Text bok of Medical Physiology, Philadelphia: Elsevier Inc, 2006

[5] R. G. Rasika Burte, "Advances In QRS detection : Modified Wavelet Energy Gradient Method," International Journal of Emerging Trends in Signal Processing, vol. I, no. I, p. 23, 2012

[6] "Physionet.org,"[Online]. Available: http://www.physionet.org/physiobank/database/mitdb/ index.html 